ACTA

AMAZONICA

\title{
Espécie nova de Euphyllodromia Shelford, 1908 do estado de Rondônia, Brasil e nova ocorrência de $E$. jugata Rehn, 1928 (Blattaria, Blattellidae)
}

Sonia Maria LOPES${ }^{1}$, Edivar Heeren de OLIVEIRA', Maria Carmosina de ARAÚJO²

RESUMO

Euphyllodromia rondonensis sp. nov. é descrita do Estado de Rondônia, Brasil, com base na genitália do macho. É assinalada uma nova ocorrência para E. jugata Rehn, 1928, coletada em ninho de vespas no estado do Acre.

PALAVRAS-CHAVE

Blattaria, E. rondonensis sp. nov., E. jugata, Rondônia, Taxonomia

\section{New species of Euphyllodromia Shelford, 1908 from Rondônia State, Brazil and new occurrence for E. jugata Rehn, 1928 (Blattaria, Blattellidae)}

ABSTRACT

Euphyllodromia rondonensis, sp. nov. is described from the Rondônia State, Brazil, based on the male genitalia. It's assinalated the new occurrence for E. jugata Rehn, 1928 from Acre State collected in wasps nest.

KEYWORDS

Blattaria, E. rondonensis sp. nov, E. jugata, Rondônia, Taxonomy.

1 Departamento de Entomologia, Museu Nacional, UFRJ, Quinta da Boa Vista, São Cristóvão, RJ, Brasil, CEP 20940-04. e-mail: sonialf@acd.ufrj.br

2 Instituto Nacional de Pesquisas da Amazônia, Manaus, AM, Brasil CEP 69011-970. e-mail: carmo@inpa.gov.br 


\section{INTRODUÇÃO}

Euphyllodromia foi proposto por Shelford (1908) na categoria de subgênero de Pseudophyllodromia Brunner, 1865 , com base na configuração das veias das tégminas. Hebard (1920) elevou-o à categoria genérica designando Euphyllodromia angustata (Latreille, 1807) para espécie-tipo.

Os espécimes de Euphyllodromia possuem tamanho pequeno; intensa pigmentação; pernas com a face ânteroventral do fêmur anterior com espinhos fortes e robustos na metade basal, seguidos de uma série de espinhos menores e esclerotinizados, terminando com três espinhos fortes e desenvolvidos; pulvilos somente no quarto tarsômero; arólios e unhas simples (Rocha e Silva, 1984).

Estão registradas para o gênero 29 espécies distribuídas do sudeste dos Estados Unidos até a região norte do Brasil.

Para a observação das peças genitais foram utilizadas técnicas para dissecação, descritas em Lopes \& Oliveira (2000). A designaçãa das peças genitais e a classificação taxonômica seguiram Mckittrick (1964).

\section{Euphyllodromia rondonensis, sp. nov.}

(Figuras 1-8, 17)

Coloração geral castanho-brilhosa. Pronoto com detalhes castanho-escuros no disco central. Cabeça castanha, clareando em direção ao clípeo. Olhos escuros com entorno delineado amarelo-claro. Antenas escuras com a base clara e leitosa. Palpos com região ventral levemente mais escura. Tégminas e asas com as veias e micropontos castanho-escuros espalhados por toda a superfície. Pernas castanho-claras com o dorso dos fêmures, base e ápice dos espinhos das tíbias, ápice dos tarsos, das unhas e base dos arólios, castanhos mais escuros.

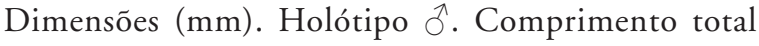
13,0; comprimento do pronoto 3,0; largura do pronoto 4,0; comprimento da tégmina 11,5; largura da tégmina 3,0.

Cabeça triangular, alongada e relativamente grande. Vértice totalmente exposto. Espaço interocular amplo, medindo cerca de três quartos da área que separa as bases das inserçôes antenais. Antenas longas, atingindo em comprimento a extremidade do abdome. Palpos maxilares com primeiro e segundo segmentos pequenos, terceiro segmento do tamanho do espaço interocular; quarto segmento do tamanho da área que separa as bases das inserções antenais, quinto segmento, dilatado e bastante tomentoso, menor em comprimento que o terceiro segmento. Tórax com pronoto curto e alargado, transverso, com entorno da base e do ápice sinuoso, abas laterais amplas com entorno lateral circular. Pernas longas e afiladas. Fêmur anterior com seis espinhos de tamanho médio a grande, da base até a região mediana, na face ânteroventral, seguidos por uma série de doze espinhos pequenos até próximo ao ápice e três espinhos distribuídos apicalmente

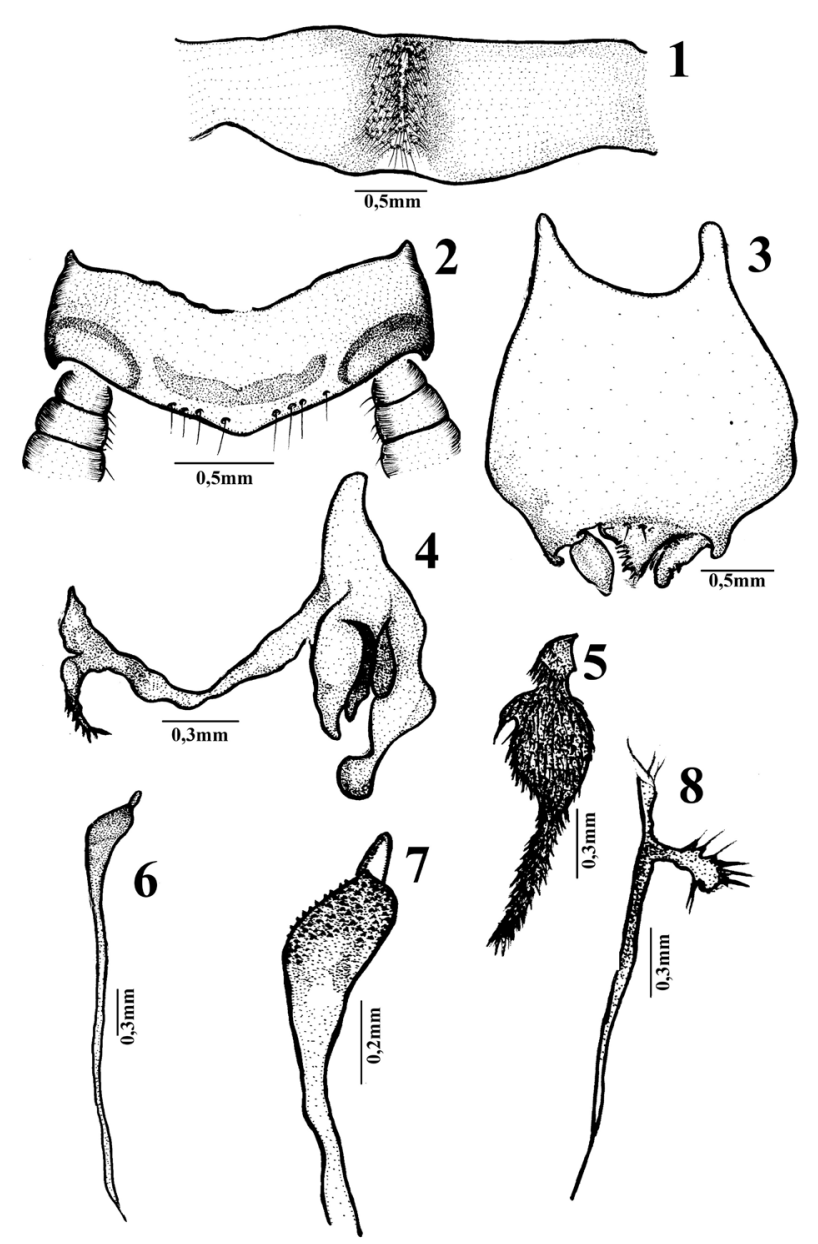

Figuras 1-8 - Euphyllodromia rondonensis, sp. n. (Holótipo đ̃). 1, modificação tergal no $7^{\circ}$ segmento; 2 , placa supra anal, vista dorsal; 3 , placa subgenital, vista ventral; 4 , falômero esquerdo, vista dorsal; 5 e 8, escleritos do falômero direito, vista dorsal; 6 , esclerito mediano, vista dorsal; 7 , ápice do esclerito mediano, vista dorsal.

(tipo B); face póstero-ventral com seis espinhos médios, sendo um deles apical. Tíbias espinhosas. Tarsos com artículo basal ultrapassando em tamanho a soma dos demais artículos. Pulvilos somente no quarto tarsômero da perna posterior que demonstra pequena projeção esclerotinizada. Unhas simétricas e sem especialização aparente. Arólio pouco desenvolvido. Tégminas longas ultrapassando o ápice dos cercos. Campo marginal levemente côncavo curto e bem marcado; campo escapular convexo; campo discoidal com veias longitudinais, apresentando numerosas veias transversais, formando uma espécie de retículo; campo anal amplo com cinco a seis veias axilares. Asas bem desenvolvidas, ramificaçôes da veia radial bastante dilatadas apicalmente. Abdome com modificação tergal no sétimo segmento concentrada medianamente na forma de fileiras de cerdas simétricas (Figura 1). Placa supra-anal levemente projetada medianamente (Figura 2). Cercos ciliados. Placa subgenital oblonga com projeçôes 


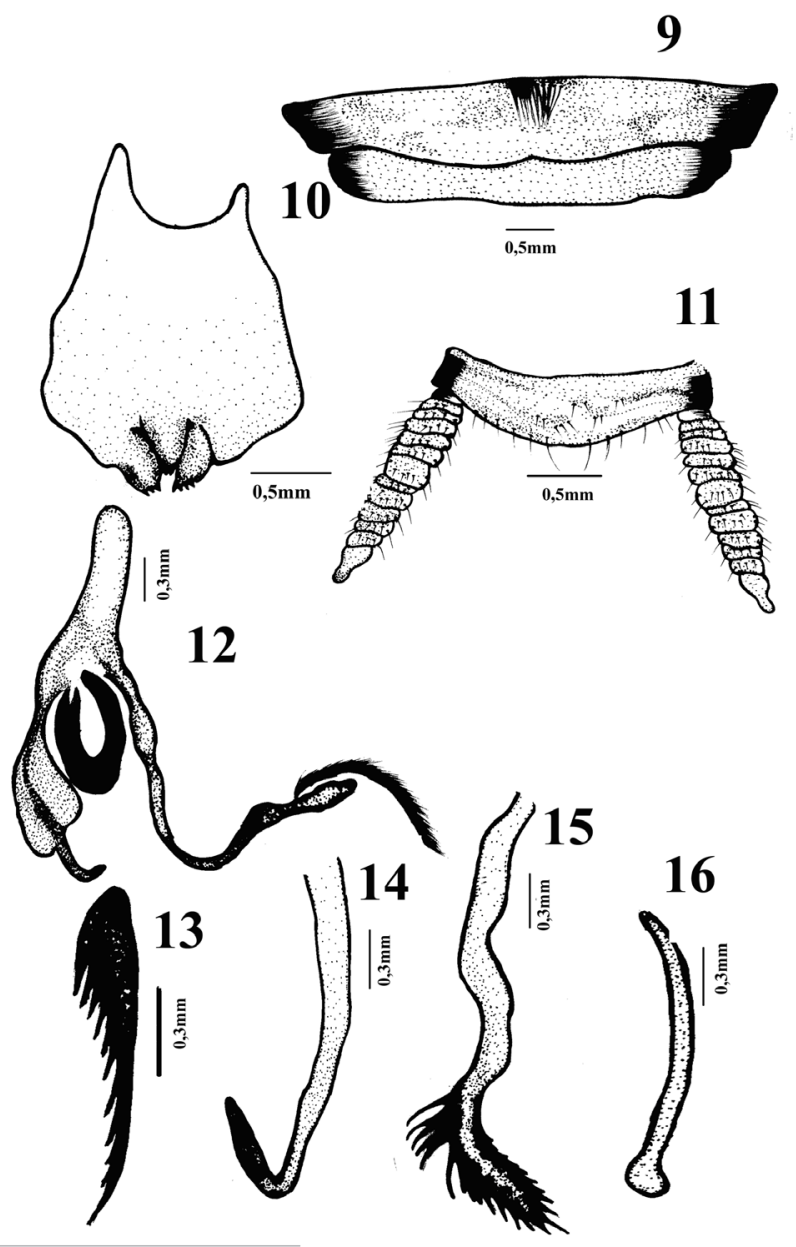

Figuras 9-16 - Euphyllodromia jugata Rehn, 1928 (ठ̋). 9, modificação tergal no $7^{\circ}$ segmento, vista dorsal; 10 , placa subgenital, vista ventral; 11 , placa supra-anal, vista dorsal; 12 , falômero esquerdo, vista dorsal; 13 e 16, escleritos do falômero direito, vista dorsal; 14 , falômero direito, vista dorsal; 15 , esclerito mediano (L2vm), vista dorsal.

laterais arredondadas, ápice com projeção mediana aguda apresentando cílios em uma das laterais. Estilos piriformes levemente assimétricos (Figura 3). Esclerito mediano afilado, com diminutos espinhos terminando em uma projeção acuminada nas extremidades (Figuras 6 e 7). Escleritos afilados com projeçôes estrelares (Figura 5 e 8); falômero esquerdo com duas projeções assimétricas, sendo uma delas menor e arredondada e a outra disforme e ciliada, internamente esclerotinizada (Figura 4).

Material examinado: Holótipo $\widehat{C}$. BRASIL: Rondônia, Porto Velho, BR-364, km 20-30, 24/X/1980 (MNRJ).

Diagnose: Espécie similar à E. amazonensis Rocha e Silva, 1984 na coloração do pronoto, dela diferindo pela configuração das placas genitais.

Etimologia: O nome da espécie é alusivo ao Estado onde o exemplar foi coletado.

\section{Euphyllodromia jugata Rehn, 1928}

(Figuras 9-16, 18)

Euphyllodromia jugata Rehn, 1928: 143, pl. XVIII, fig. 6; Rocha e Silva 1984: 76.

Holótipo $\widehat{0}$ - BOLÍVIA, Província Sara (Coleção Hebard, tipo no 1.124 na Academy of Natural Sciences of Philadelphia.

Distribuição: Bolívia, Brasil (Acre, Amazonas,
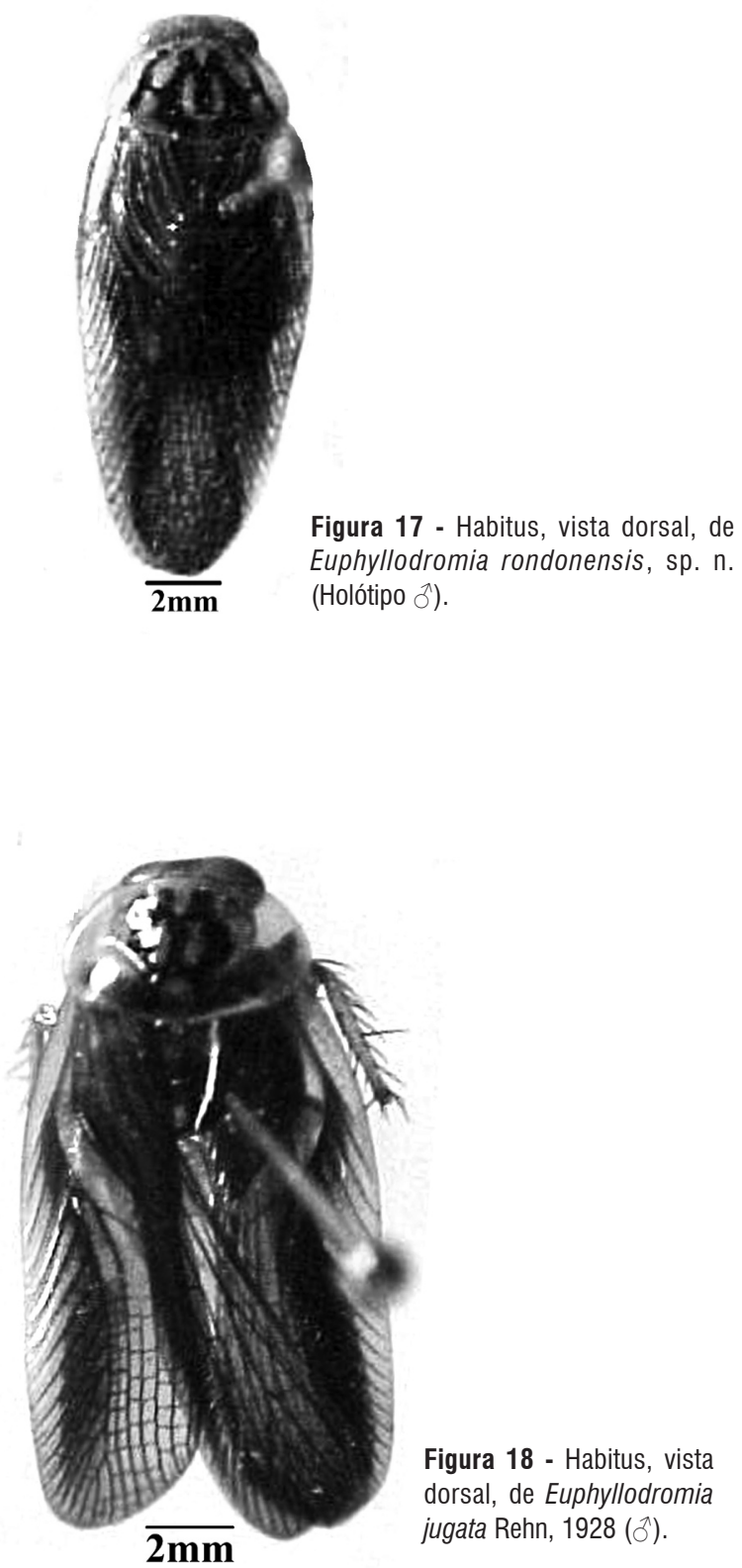

Figura 18 - Habitus, vista dorsal, de Euphyllodromia jugata Rehn, 1928 (ठ̋). 


\section{Rondônia).}

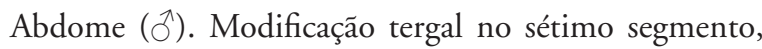
ressaltada pela presença de um grupo de cerdas longas na região mediana do mesmo (Figura 9). Placa supra-anal curta, ciliada, e levemente prolongada entre os cercos, os quais são robustos e alongados (Figura 11). Placa subgenital assimétrica afunilando para o ápice, com uma região distinta com espinhos entre os estilos. Estilos lamelares e ciliados (Figura 10). Falômero esquerdo em forma de $\mathrm{Y}$ invertido com projeçōes assimétricas, sendo uma delas muito prolongada; estrutura mediana arqueada e esclerotinizada (Figura 12). Falômero direito em gancho (Figura 14). Escleritos do falômero direito diferenciados (Figuras 13 e 16). Esclerito mediano em forma de estilete, muito afilado e sinuoso com projeções ciliares látero-apicais (Figura 15).

Material examinado: BRASIL, Acre, Senador Guiomard, Reserva Catuaba, 1004'S 67036'W, Elder F. Morato col. (ninho 506/2), 2/X/2001 (ठ)); Amazonas, Manaus, Reserva Ducke, Oliveira col., XI/1966 (ㅇ); Amazonas: Tiguda, José

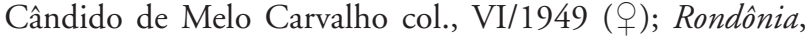
Porto Velho, Rio Guaporé, M. Alvarenga col., XII/1954 (2 ठ) (MNRJ).

Bionomia. E. jugata foi coletada em ninho de vespas família Sphecidae, do gênero Podium Fabricius, 1804. São utilizadas como recurso alimentar para as larvas.

\section{AGRADECIMENTOS}

Ao Conselho Nacional de Desenvolvimento Científico e Tecnológico (CNPq) e Fundação de Amparo à Pesquisa (FAPERJ); à Dra. Janira Martins Costa, professora do Museu Nacional, pelo apoio técnico; ao Dr. Elder Ferreira Morato (UFMG), pela coleta do material enviado para identificação.

\section{BIBLIOGRAFIA CITADA}

Hebard, M. 1920. The Blattidae of Panama. Memoirs of the American Entomological Society, 4: 1-148.

Lopes, S.M; Oliveira, E.H. 2000. Espécie Nova de Eublaberus Hebard, 1919 do Estado de Goiás, Brasil e notas sobre E. marajoara Rocha e Silva-Albuquerque, 1972 (Blaberidae, Blaberinae). Boletim do Museu Nacional, 433: 1-5.

Mckittrick, F.A. 1964. Evolutionary studies of cockroaches. Cornel University Agricultural Experiment Station Memoir, 389: 1-197.

Rehn, J.A.G. 1928. New or little known Neotropical Blattidae (Orthoptera). Number one. Transactions American Entomological Society Philadelphia, 54: 125-194.

Rocha e Silva, I. 1984. Revisão do gênero Euphyllodromia Shelford, 1908 (Blattellidae: Blattodea: Dictyoptera). Revista Brasileira de Entomologia, 28(1): 65-85.

Shelford, R. 1908. Orthoptera. Fam. Blattidae. Subfam. Phyllodromiinae. Genera Insectorum, 73: 1-29.

Recebido em 07/12/2006

Aceito em 23/03/2007 